\title{
Crossover between Monopole and Multipole Plasmon of Cs Monolayers on Si(111) Individually Resolved in Energy and Momentum
}

\author{
Volkmar Zielasek, Neele Rönitz, ${ }^{*}$ Martin Henzler, and Herbert Pfnür \\ Institut für Festkörperphysik, Universität Hannover, Appelstr. 2, 30167 Hannover, Germany
}

(Received 18 January 2006; published 16 May 2006)

\begin{abstract}
The evolution of the plasmon spectrum of the $\mathrm{Si}(111)(7 \times 7)$-Cs surface has been studied by energy loss spectroscopy individually resolved in energy and momentum during the transition from substrate to Cs overlayer metallization. The multipole plasmon is identified by an extremely narrow angular distribution of the inelastic electron scattering, unaccounted for by standard dipole scattering theory. A crossover between multipole and monopole surface plasmon is observed at finite surface wave vectors $q_{\|}$, depending on Cs coverage, and reveals a high sensitivity of the short-wavelength multipole components on surface morphology.
\end{abstract}

DOI: 10.1103/PhysRevLett.96.196801

PACS numbers: 73.20.Mf, 61.14.Hg, 68.49.Jk

Understanding the spectrum of surface plasmon eigenmodes and their dispersion is fundamental to understanding the physics and chemistry at metallic surfaces, because plasmons sensitively depend on the free electron density and distribution within the surface. Therefore, they have been extensively studied at alkali metal (AM) surfaces and AM layers on metal substrates [1-5] in order to test the validity of surface models and approximations. Despite the progress that has been made [see Refs. [6,7] for reviews ], the understanding of the collective excitations of ultrathin metallic layers is still incomplete. In particular, a comparison of calculations and experimental results obtained for ultrathin AM layers on metal substrates has been inconclusive, in part probably due to the limitations of standard electron energy loss spectroscopy (EELS) and photoyield measurements [5]. This Letter will demonstrate experimental results which overcome previous limitations and reveal that theoretical models need to be refined in order to account for effects of surface geometry and morphology on the plasmon spectrum in the limit of ultrathin layers.

In EELS, the ordinary monopole surface plasmon (SP) [8] is a well-known feature due to intense dipole scattering [9]. Multipole surface plasmon (MP) modes, however, already predicted by hydrodynamic models of the electron gas several decades ago [10,11], have so far been unequivocally identified only by relatively weak impact scattering features in EELS at thick AM layers on aluminum substrates $[2,12]$ and by photoyield measurements, which are restricted to $q_{\|} \leq \omega / c$, with $q_{\|}$as wave vector, $\omega$ as frequency, and $c$ as the velocity of light. In the nonretarded limit, i.e., for $q_{\|}>\omega / c$, MP modes are not expected to set up long-range dipole fields in the vacuum above the surface, which makes them hard to detect for EELS. Consequently, EELS data obtained for thin AM layers on metal surfaces $[3,13]$ were dominated by scattering at the SP even for $q_{\|}=0$, where calculations and photoyield measurements only show the MP and the AM bulk plasmon (BP) $[4,5,14]$.
Using a unique experimental setup for high-resolution studies of the angular distribution of inelastic electron scattering, we have identified SP, MP, and BP, individually resolved in energy and momentum, during the transition of $\operatorname{Si}(111)(7 \times 7)$-Cs from initial Cs adsorption to a saturated pseudomorphic Cs wetting layer. For electron scattering at the MP mode we find a characteristic narrow angular scattering profile, unaccounted for by standard dipole scattering theory. The dominant plasmon evolves from SP to MP while the Cs coverage is increased from 1 to 3 physical layers [PL, 1 PL corresponding to the saturation coverage at room temperature which is $\approx 25 \mathrm{Cs}$ atoms per $7 \times 7$ unit cell [15]].

In an intuitive picture laid out by Liebsch, the MP of an AM layer is formed by coupling of the monopole plasmons of the AM-substrate interface and the AM surface [7]. Since the SP amplitudes decay as $\exp \left(-q_{\|} z\right)$, with $z$ as distance from the interface or surface, respectively, the short-wavelength components of the MP mode are expected to be efficiently damped only as the layer thickness exceeds $2 \pi / q_{\|}$, the surface component of the plasmon wavelength. We will show that also in the limit of ultrathin AM layers a crossover between SP and MP is observed at finite $q_{\|}$, shifting to larger wave vectors as the Cs coverage is increased. Complementary low-energy electron diffraction (LEED) and measurements of the surface sheet conductance reveal correlations of the AM layer morphology and the evolution of the surface plasmon spectrum which have so far not been accounted for by theory or experiment.

Our experiments have been performed in ultrahigh vacuum (base pressure below $10^{-8} \mathrm{~Pa}$ ) using an ELS-LEED system [16], which may be considered either as LEED with high energy resolution or as EELS with high momentum resolution (experimentally limited by the quality of the sample surface, best achieved resolution $0.04 \mathrm{~nm}^{-1}$ ). The scattering geometry is near normal incidence and detection (specular reflection occurs at $\Theta_{i}=6^{\circ}$ ), in contrast to conventional EELS. Monochromator and analyzer were 
tuned for high transmission, restricting the energy resolution to $60 \mathrm{meV}$ full width at half maximum (FWHM). The primary electron energy was $47 \mathrm{eV}$ for all experimental data presented in the following.

Substrate samples were cut from a phosphorus-doped wafer of high resistivity ( $>1000 \Omega \mathrm{cm}$ ) and mounted on a stage for liquid nitrogen cooling. Clean $\operatorname{Si}(111)(7 \times 7)$ surfaces were prepared by repeated rapid direct current heating to $1150^{\circ} \mathrm{C}$ in order to remove the protective surface oxide, while keeping the ambient pressure below $2 \times$ $10^{-7} \mathrm{~Pa}$. The surface quality was monitored by LEED. The setup is complemented by four-probe measurements of the electrical surface sheet conductance, for which direct current was sent through tantalum clamps fixed at both ends of the sample. In the center two Ta tips were pressed onto the substrate by a springy metal wire for voltage measurements. Cs was deposited from well-outgassed chromate dispensers (SAES getters) keeping the pressure in the $10^{-8} \mathrm{~Pa}$ regime. The deposition rate was calibrated by the onset of significant sheet conductance in the $10^{-4} \Omega^{-1}$ regime which had been demonstrated to occur at 2.2 PL coverage [17].

On the $\mathrm{Si}(111)(7 \times 7)$ surface, Cs initially adsorbs at adatom and restatom sites $[18,19]$ within the faulted and unfaulted halves of the dimer-adatom-stacking fault (DAS) reconstruction of the substrate [20]. At room temperature (RT), the saturation coverage of 1 PL [15] is reached after the dangling bonds of the substrate are saturated [19,21]. At that coverage, the surface is metallic, already [22,23], exhibiting a plasmon loss in EELS. Continued growth of the Cs layer can be achieved at temperatures below $260 \mathrm{~K}$ [19]. The surface sheet conductance measured during Cs deposition at $210 \mathrm{~K}$ is shown in Fig. 1 and indicates formation of a continuous Cs wetting layer at 2.5 PL coverage. Starting from an initial Cs adsorbate layer at 0.75 PL

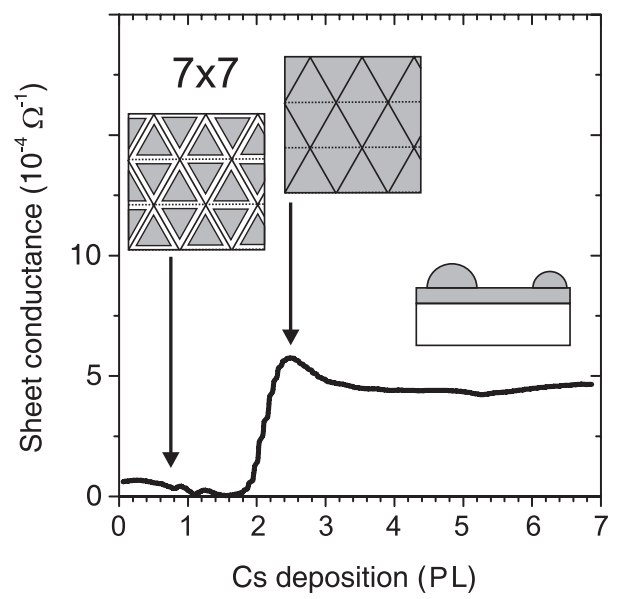

FIG. 1. Sheet conductance of Cs deposited on $\mathrm{Si}(111)-(7 \times 7)$ at $210 \mathrm{~K}$. Insets are meant as schematic representation of surface structures expected for $0.75 \mathrm{PL}$ (Cs islands on faulted and unfaulted halves of the DAS substrate reconstruction), 2.5 PL (continuous Cs wetting layer), and coverages above 2.5 PL (Cs clusters on top of wetting layer), respectively. which is made of Cs islands within the $(7 \times 7)$ substrate unit cells, the wetting layer formation probably starts by interconnecting these monolayer high islands. Upon continued deposition beyond 2.5 PL, neither EELS nor LEED, showing a $(7 \times 7)$ surface reconstruction, significantly change, and the sheet conductance saturates at a value $20 \%$ below maximum, an indication for the formation of three-dimensional (3D) islands that are electrically not connected.

EEL spectra recorded for three different amounts of Cs deposited on $\operatorname{Si}(111)(7 \times 7)$ at a temperature of $210 \mathrm{~K}$ (Fig. 2) reveal a shift of the plasmon frequency at $q_{\|}=0$, as mentioned by Weitering et al. [22], during the evolution of the surface from Cs-modified substrate states to a Cs metal layer. The shift probably reflects an increase of the effective electron density in the metallic surface state or layer. As shown later, we can identify the energy loss peak observed at $0.94 \mathrm{eV}$ for $1 \mathrm{PL}$ coverage as SP loss and the peaks at $1.68 \mathrm{eV}$ (1.4 PL) and $2.16 \mathrm{eV}$ (6.2 PL), respectively, as MP losses. Up to $2.8 \mathrm{ML}$, the MP loss continuously shifts to higher frequencies with increasing amount of deposited Cs. The maximum energy of $2.2 \mathrm{eV}$ is reached right after the jumplike increase of the surface sheet conductance up to $6 \times 10^{-4} \Omega^{-1}$ is observed. For comparison, the MP energy of Cs layers on $\mathrm{Al}(111)$ was determined as $2.4 \mathrm{eV}$ [12]. The shoulder on the high-energy side of the MP peak at 6.2 PL in Fig. 2 is due to the BP at $2.7 \mathrm{eV}$.

To distinguish between SP and MP, we have measured profiles of the inelastic electron scattering at the plasmon with respect to the surface component of the scattering vector $\Delta K_{\|}=-q_{\|}$(Fig. 3). The top panel (I) shows the measured profile of the $0.94 \mathrm{eV}$ energy loss observed for 1 PL coverage [see also (I) in Fig. 2]. It is as expected for scattering at the SP mode of an ultrathin metallic overlayer because the measurement is perfectly fitted by the dipole scattering lobe of a thin dipole-active surface layer, i.e., a

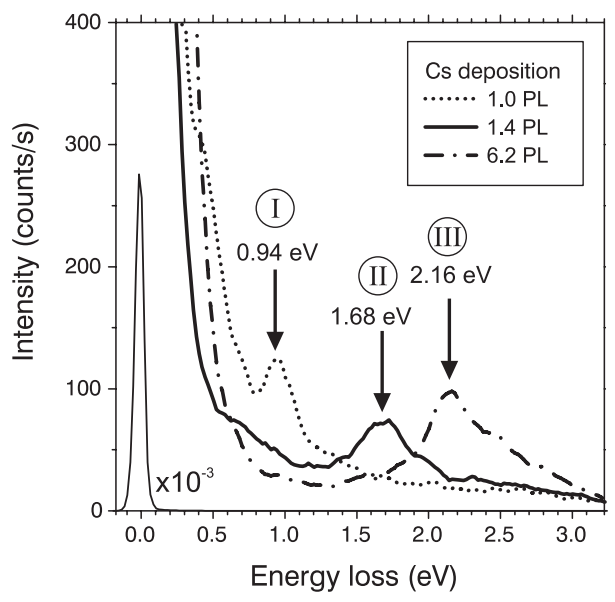

FIG. 2. Electron energy loss spectra for $\Delta K_{\|}=0$ for three different amounts of $\mathrm{Cs}$ deposited on $\mathrm{Si}(111)-(7 \times 7)$ at $210 \mathrm{~K}$. The arrows mark loss energies due to surface plasmon scattering for which scattering profiles have been measured (see Fig. 3). 


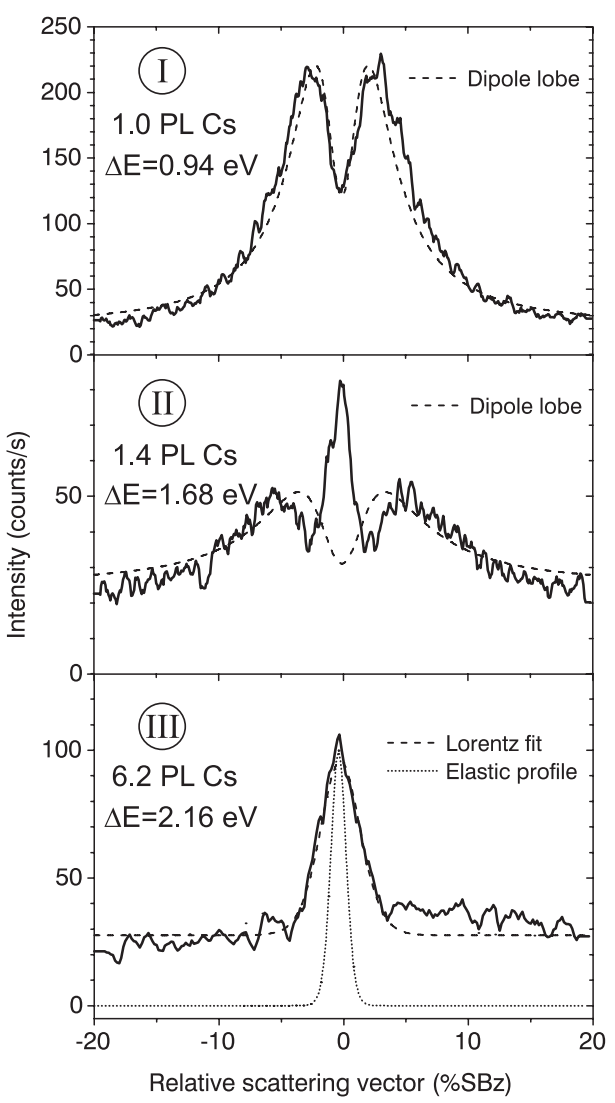

FIG. 3. Scattering profiles with respect to $\Delta K_{\|}$for inelastic scattering at $\mathrm{Cs} / \mathrm{Si}(111)$. The scattering vector $\Delta K_{\|}$is given in $\%$ of the distance between the (00) and the (10) diffraction spot of the $\mathrm{Si}(111)$ surface. $10 \% \mathrm{SBz}$ corresponds to $0.188 \AA^{-1}$.

cross section $d S / d \Delta K_{\|}$proportional to $\left(\Delta K_{\|}\right)^{2} /\left[(\omega / v)^{2}+\right.$ $\left.\left(\Delta K_{\|}\right)^{2}\right]^{2}$ [9] (with $v$ as electron velocity), which is shown in the upper panel of Fig. 3 after convolution with the elastic profile. The elastic profile, depicted in the bottom panel with normalized intensity, represents the momentum resolution which is limited by the crystalline quality of the sample surface.

The bottom panel (III) of Fig. 3 shows the measured profile of the $2.2 \mathrm{eV}$ loss observed after deposition of 6.2 PL Cs. In contrast to (I), it is dominated by a narrow peak at $\Delta K_{\|}=0$, which has, to our knowledge, not been reported for standard reflection EELS before. The profile indicates a strong forward scattering mechanism which is unexpected for the statistics of localized impact scattering events. At the same time, the profile is not represented by available results of classic dipole scattering theory [9], even if effects of retardation are taken into account [24]. Given the energy loss alone, it appears compelling to ascribe the peak observed in panel (III) to scattering at the MP mode of the Cs overlayer. The narrow distribution of the scattering around $q_{\|}=0$ indicates that dipole scattering selection rules [9] do not apply and scattering at electromagnetic radiation of the radiative branch of the MP $\left(q_{\|} \leq \omega / c\right)$ may be involved.
A crossover between MP and SP can be identified in the scattering profile of the $1.65 \mathrm{eV}$ loss for $1.4 \mathrm{PL}$ Cs coverage, shown in panel (II). The profile exhibits a central spike at $\Delta K_{\|}=0$ due to MP scattering. Additionally, a broad symmetric shoulder, very likely due to dipole scattering at an ordinary SP mode, appears. A comparison of the shoulder with the expected dipole lobe reveals that the experimental profile is broadened, indicating that for small wave vectors the surface plasmon has switched from SP to MP. Obviously SP and MP do not coexist at $q_{\|}=0$, in accordance with predictions by Liebsch [25]. With increasing Cs coverage up to 2.8 PL, further suppression of the SP scattering is observed. Beyond 2.8 PL, after a continuous Cs wetting layer has formed, only the central spike is detected, on top of a constant background present in all profiles of Fig. 3.

The crossover is shown in detail in Fig. 4 at a concentration of 2.8 PL, where we plot the loss intensity for various $q_{\|}$as a function of loss energy. While the SP is suppressed for small plasmon wave vectors, it is still present for large wave vectors, even on a continuous 2.8 PL thick Cs wetting layer, as the energy loss spectra for different $q_{\|}$demonstrate. The inset shows the center positions of Lorentzian profiles fitted to the spectra. For small wave vectors, a shoulder on the high-energy side of the MP peak indicates scattering due to the Cs bulk plasmon (BP), in correspondence to calculations by Liebsch [5]. At $q_{\|}=0$ the energies of MP and BP are $2.2 \mathrm{eV}$ and $2.7 \mathrm{eV}$, respectively. For $q_{\|}>0.1 \AA^{-1}$ the MP disappears and a lower energy loss at $1.9 \mathrm{eV}$, representing the SP, is detected. With respect to their ratios, there is a perfect match between the experimental mode energies and theory, which predicts $\omega_{\mathrm{MP}}=0.8 \omega_{\mathrm{BP}}$ and $\omega_{\mathrm{SP}}=\omega_{\mathrm{BP}} / \sqrt{2}$. The perfect match indicates that we have resolved scattering at the SP, MP, and BP individually in energy and momentum. The wave vector of crossover between SP and MP depends

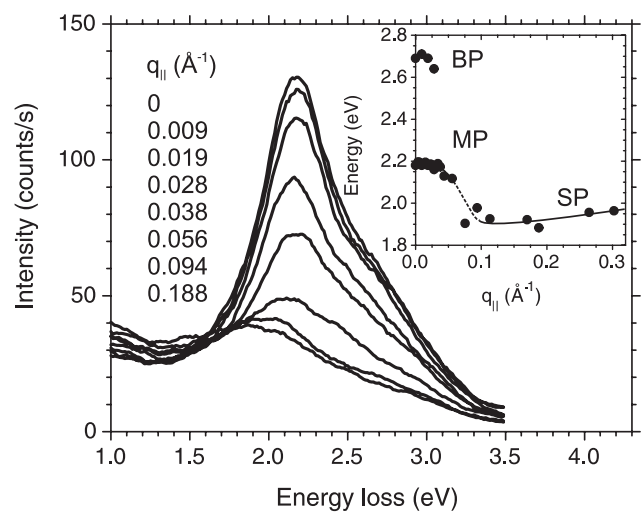

FIG. 4. Plasmon energy loss spectra of a Cs wetting layer $(2.8 \mathrm{PL})$ on $\mathrm{Si}(111)(7 \times 7)$ for different wave vectors $q_{\|}$. Inset shows the energies of the surface plasmons (MP, SP) and the bulk plasmon (BP) of the Cs layer, as determined from fits to the measured spectra. The line indicating dispersion of the combined MP-SP modes is meant as guide to the eyes. 


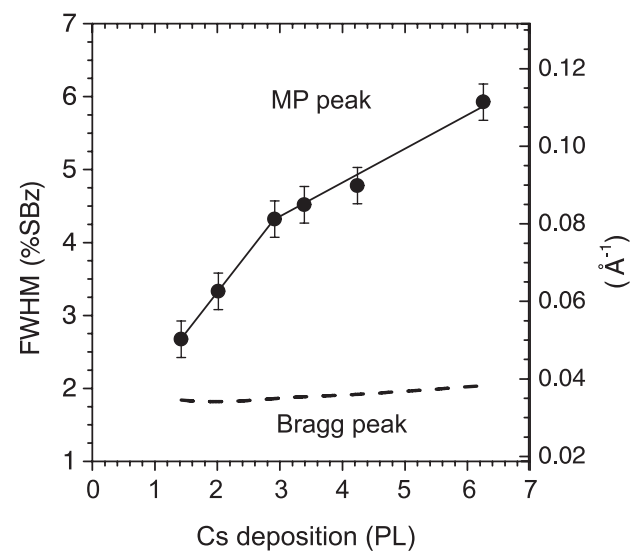

FIG. 5. FWHM of the multipole plasmon peak during Cs deposition at $210 \mathrm{~K}$. The solid lines are meant as guide to the eyes. The dashed curve shows the FWHM of the elastic specular Bragg peak.

on Cs coverage. As a measure we take the full width at half maximum (FWHM) of the MP peak in the inelastic scattering profiles which is shown in Fig. 5 for increasing amount of deposited Cs. Even up to completion of the wetting layer, the FWHM of the MP increases for coverages from 1.4 to $2.8 \mathrm{PL}$, in contrast to the expectations from Ref. [7], whereas for thicker layers the further increase can be understood as being due to scattering from 3D islands. The change of slope at about 2.8 PL Cs deposition shows that the shift of the crossover between SP and MP sensitively reflects the evolution of the surface from the initial adsorbate layer to a complete Cs wetting layer.

In conclusion, our correlation of EELS, structural and conductance data shows that the dominant surface plasmon mode of ultrathin Cs layers on $\mathrm{Si}(111)$ evolves from SP to MP only as the Cs coverage is increased beyond the saturation coverage at RT but before the surface has transformed from adsorbate-modified surface states to a continuous metallic AM wetting layer. While the SP mode is clearly identified by a dipole scattering profile in EELS, the narrow MP peak at $q_{\|}=0$ indicates a strong inelastic forward scattering mechanism which cannot be accounted for by classic dipole scattering and may be special for multipole excitations of thin films. Since the MP peak has not been observed in ordinary EELS, the unique scattering geometry of our experiments may be a prerequisite. By this Letter, we hope to stimulate further theoretical and experimental efforts with respect to plasmons of ultrathin metal layers. Upon Cs deposition, the MP forms first in the limit $q_{\|} \rightarrow 0$, while the conductivity data clearly show that the wetting layer is far from continuous on the long range. An intuitive picture for this discrimination between longand short-wavelength components, as it exists in the limit of thick AM layers [7], is missing and calls for refinements of the theoretical models.
Financial support by Deutsche Forschungsgemeinschaft is gratefully acknowledged.

*Present address: Philips Semiconductors, Stresemannallee 101, 22529 Hamburg, Germany.

[1] A. G. Eguiluz and D. A. Campbell, Phys. Rev. B 31, 7572 (1985).

[2] K. D. Tsuei, E. W. Plummer, A. Liebsch, K. Kempa, and P. Bakshi, Phys. Rev. Lett. 64, 44 (1990).

[3] J.A. Gaspar, A. G. Eguiluz, K.D. Tsuei, and E.W. Plummer, Phys. Rev. Lett. 67, 2854 (1991).

[4] S. R. Barman, C. Stampfl, P. Häberle, W. Ibanez, Y. Q. Cai, and K. Horn, Phys. Rev. B 64, 195410 (2001).

[5] A. Liebsch, B. O. Kim, and E. W. Plummer, Phys. Rev. B 63, 125416 (2001).

[6] M. Rocca, Surf. Sci. Rep. 22, 1 (1995).

[7] A. Liebsch, Electronic Excitations at Metal Surfaces (Plenum, New York, 1997).

[8] R. H. Ritchie, Phys. Rev. 106, 874 (1957).

[9] H. Ibach and D.L. Mills, Electron Energy Loss Spectroscopy and Surface Vibrations (Academic, New York, 1982).

[10] A. J. Bennett, Phys. Rev. B 1, 203 (1970).

[11] A. Eguiluz, S. C. Ying, and J. J. Quinn, Phys. Rev. B 11, 2118 (1975).

[12] K. D. Tsuei, E. W. Plummer, A. Liebsch, K. Kempa, and P. Bakshi, Surf. Sci. 247, 302 (1991).

[13] G. Chiarello, A. Cupolillo, A. Amoddeo, L. S. Caputi, L. Papagno, and E. Colavita, Phys. Rev. B 55, 1376 (1997).

[14] S. R. Barman, K. Horn, P. Häberle, H. Ishida, and A. Liebsch, Phys. Rev. B 57, 6662 (1998).

[15] W. B. Sherman, R. Banerjee, N. J. DiNardo, and W. R. Graham, Surf. Sci. 479, 136 (2001).

[16] H. Claus, A. Büssenschütt, and M. Henzler, Rev. Sci. Instrum. 63, 2195 (1992).

[17] The amount of Cs was determined by collecting and electrically counting Cs ions, thermally desorbed from a $\mathrm{Pt}$ foil of known surface area close to the Si sample position. Because of the high $\mathrm{Pt}$ work function it is assumed that the ionization rate of desorbed Cs is close to 1 (E. Suliga, Diploma thesis, University of Hannover, 1977).

[18] T. Hashizume, K. Motai, Y. Hasegawa, I. Sumita, H. Tanaka, S. Amano, S. Hyodo, and T. Sakurai, J. Vac. Sci. Technol. A 9, 745 (1991).

[19] K. Pedersen, P.E. Hansen, and P. Morgen, Phys. Status Solidi A 170, 411 (1998).

[20] K. Takayanagi, Y. Tanishiro, M. Takahashi, and S. Takahashi, J. Vac. Sci. Technol. A 3, 1502 (1985).

[21] K. O. Magnusson, S. Wiklund, R. Dudde, and B. Reihl, Phys. Rev. B 44, 5657 (1991).

[22] H.H. Weitering, J. Chen, R. Perez-Sandoz, and N.J. DiNardo, Surf. Sci. 307-309, 978 (1994).

[23] K. D. Lee, J. R. Ahn, and J. W. Chung, Appl. Phys. A 68, 115 (1999).

[24] Y. Uehara, Y. Suzuki, and S. Ushioda, J. Phys. Soc. Jpn. 69, 2675 (2000).

[25] A. Liebsch, Phys. Rev. Lett. 67, 2858 (1991). 